\title{
Método de ressonância para predição das propriedades mecânicas das madeiras de Eucalyptus urophylla e Pinus oocarpa termorretificadas
}

\section{Resonance method for predicting the mechanical properties of heat-treated Eucalyptus urophylla and Pinus oocarpa wood}

\author{
Amélia Guimarães Carvalho ${ }^{1}$, Antonio José Vinha Zanuncio ${ }^{2}$, \\ Carlos Miguel Simões da Silva ${ }^{1}$, Angélica de Cassia Oliveira Carneiro ${ }^{1}$, \\ Marcos Oliveira de Paula ${ }^{1}$
}

\author{
${ }^{1}$ Laboratório de Painéis e Energia da Madeira - LAPEM/UFV CEP: 36570-000 Viçosa, MG. \\ e-mail: ameliagcarvalho@gmail.com, karmiguel@yahoo.com.br, cassiacarneiro1@gmail.com, modep@ufv.br \\ ${ }^{2}$ Unicentro - BR $153 \mathrm{Km} 7$ Bairro riozinho - CEP 84500-000, Irati, PR \\ e-mail: ajvzanuncio@yahoo.com.br
}

\begin{abstract}
RESUMO
Vibrações transversais apresentam potencial para avaliação da madeira devido a alta precisão, baixo custo e rapidez, mas sua aplicação em madeira termorretificada ainda é pouco estudada. O objetivo deste trabalho foi avaliar as propriedades físicas e mecânicas de madeira termorretificada de Eucalyptus urophylla e Pinus oocarpa e relacionar a análise de ressonância (Bing) com o modulo de elasticidade estático e de ruptura da madeira.Corpos de prova foram confeccionados de três árvores de Eucalyptus urophylla e três de Pinus oocarpa e termorretificados a 150,175 , 200 e $225^{\circ} \mathrm{C}$, com taxa de aquecimento de $5^{\circ} \mathrm{C} / \mathrm{min}$, tempo de residência de quatro horas com pressão atmosférica e presença de ar.

A termorretificação reduziu a densidade básica, inchamento volumétrico, inchamento linear, coeficiente de anisotropia, umidade de equilíbrio e resistência mecânica da madeira de ambas as espécies. A análise de ressonância (Bing) apresentou alta relação com o modulo de elasticidade estático e o módulo de ruptura da madeira termorretificada de ambas as espécies e, portanto, esta técnica pode ser utilizada para avaliar as propriedades mecânicas da madeira submetidas a este processo.
\end{abstract}

Palavras-chave: Bing, Eucalyptus urophylla; Pinus oocarpa; Tratamento térmico.

\begin{abstract}
The analysis of the transverse vibrations has the potential to evaluate the wood due to its high precision, low cost and quickly, however there are few studies about its use in heat treated wood. The aim of this study was to evaluate the physical and mechanical properties of heat-treated Eucalyptus urophylla and Pinus oocarpa wood and relate the analysis of resonance (Bing) with the modulus of elasticity and modulus of rupture of wood. Three Eucalyptus urophylla and Pinus oocarpa trees were used and specimens were prepared and heat-treated at $150,175,200$ and $225^{\circ} \mathrm{C}$ with a heating rate of $5^{\circ} \mathrm{C} / \mathrm{min}$, four hours of residence time at atmospheric pressure and the presence of air. The heat treatment reduced the volumetric swelling, linear swelling, anisotropy factor, equilibrium moisture content and mechanical strength of the wood in both species. The resonance analysis (Bing) showed a high correlation with the static modulus of elasticity and modulus of rupture in heat-treated wood for both species, thus, this technique can be used to evaluate the mechanical properties during this process.
\end{abstract}

Keywords: Bing, Eucalyptus urophylla; Pinus oocarpa; Heat treatment.

\section{INTRODUÇÃO}

A termorretificação da madeira consiste na aplicação de calor até $260^{\circ} \mathrm{C}$ [1] em diferente duração [2,3], at- 
mosfera [4] e umidade [5]. Este processo, de fácil aplicação e sem utilização de produtos químicos [1,6], visa, principalmente, degradar os carboidratos da madeira [7], limitando a adsorção da umidade e aumentando a estabilidade dimensional [8].

A destruição dos carboidratos pela termorretificação reduz a resistência mecânica da madeira, afetando o modulo de ruptura e elasticidade [9,10]. Isto torna necessário monitorar a queda na resistência mecânica da madeira para não inviabilizar o uso da termorretificação.

Propriedades mecânicas da madeira termorretificada devem ser avaliadas com métodos rápidos, precisos e de baixo custo. A avaliação não destrutiva é uma importante ferramenta na inferência de propriedades mecânicas da madeira, devido, principalmente, ao baixo custo dos equipamentos, rapidez e praticidade dos testes. A utilização de vibrações transversais, que consiste em relacionar a frequência de vibração natural de um material com a sua resistência à flexão ou compressão. Leite [11] encontraram uma forte correlação ( $\mathrm{R}=$ 0,88 ) entre o módulo de elasticidade determinado pela vibração transversal e o valor determinado pela máquina de ensaio universal, para a madeira de Copaifera langsdorffii.

O objetivo deste trabalho foi avaliar as propriedades físicas e mecânicas de madeira termorretificada de Eucalyptus urophylla e Pinus oocarpa e relacionar a análise de ressonância (Bing) com o modulo de elasticidade estático e de ruptura da madeira.

\section{MATERIAIS E MÉTODOS}

Três árvores de Eucalyptus urophylla com 15 anos e Pinus oocarpa com 18 anos foram selecionadas. Após a colheita, um pranchão central foi removido de cada árvore e utilizado para preparo de tabuas que foram secas ao ar livre até a estabilização da massa. Em seguida, corpos de prova (amostras) com $2 \times 2 \times 3 \mathrm{~cm}$ e de $2 \times 2$ $\times 30 \mathrm{~cm}$ foram confeccionados a partir das tábuas para os testes físicos e mecânicos, respectivamente. Essas amostras foram secas em estuda a $103^{\circ} \mathrm{C}$ até a condição anidra e termorretificadas em uma autoclave a 150 , 175,200 e $225^{\circ} \mathrm{C}$ com taxa de aquecimento de $5^{\circ} \mathrm{C} / \mathrm{min}$ e tempo de residência de quatro horas sob pressão atmosférica e presença de ar.

A perda de massa das amostras de $2 \times 2 \times 3 \mathrm{~cm}$ foi calculada com a equação 1 :

$\operatorname{PM}(\%)=(((M i-M f)) / M i) \times 100$

Onde:

$\mathrm{PM}=$ perda de massa $(\%)$,

$\mathrm{Mi}=$ massa antes da termorretificação,

Mf= massa após a termorretificação.

Essas amostras foram acondicionadas em câmara climática a $23^{\circ} \mathrm{C}$ e $50 \%$ de umidade relativa até estabilização da massa. A umidade das amostras foi calculada com a equação 2:

$\mathrm{U}(\%)=(((\mathrm{Mu}-\mathrm{Ms})) / \mathrm{Ms}) \times 100$

Onde:

$\mathrm{U}(\%)=$ umidade das amostras,

$\mathrm{Mu}=$ massa úmida das amostras,

Ms= massa seca das amostras.

As amostras foram colocadas em dessecador com água acoplado a uma bomba de vácuo, e submetidas a vários ciclos até atingirem a saturação para determinação do inchamento volumétrico com a equação 3 :

$\operatorname{IV}(\%)=(((V s-V a)) / V a) \times 100$

Onde:

IV (\%)= inchamento volumétrico; 
Vs= volume da madeira saturada;

$\mathrm{Va}=$ volume da madeira anidra.

O inchamento radial foi determinado com a equação 4:

$\operatorname{IR}(\%)=(((\mathrm{CRs}-\mathrm{CRa})) / \mathrm{CRa}) \times 100$

Onde:

IR(\%)= Inchamento radial;

$\mathrm{CRs}=$ comprimento radial da madeira saturada;

$\mathrm{CRa}=$ comprimento radial no estado anidro.

O inchamento tangencial foi calculado conforme equação 5:

$\mathrm{IT}(\%)=(((\mathrm{CTs}-\mathrm{CTa})) / \mathrm{CTa}) \times 100$

Onde:

IT(\%)= Inchamento tangencial;

$\mathrm{CTs}=$ comprimento tangencial da madeira saturada;

$\mathrm{CTa}=$ comprimento tangencial no estado anidro.

O coeficiente de anisotropia foi determinado pela razão entre o inchamento tangencial e radial. A densidade básica foi calculada conforme equação 6:

$\mathrm{D}=\mathrm{M} / \mathrm{V}$

Onde:

$\mathrm{D}=$ densidade básica $\left(\mathrm{g} / \mathrm{cm}^{3}\right)$,

$\mathrm{M}=$ massa anidra da amostra (g);

$\mathrm{V}=$ volume saturado da amostra $\left(\mathrm{cm}^{3}\right)$.

O equipamento utilizado para a análise e a mensuração das frequências de vibração transversal foi o BING - Beam Identification by Nondestructive Grading. O ensaio foi realizado nos corpos de prova de $2 \times 2$ $\times 30 \mathrm{~cm}$ com os mesmos sobre um suporte elástico, sendo a vibração produzida por meio de um impacto mecânico, com a batida de um martelo em uma extremidade e o sinal é captado na outra por um microfone ligado à placa de som de um computador. Essas vibrações foram interpretadas com o programa BINGß version 9.1.3., que fornece o modulo de elasticidade dinâmico da madeira [11]. Os ensaios destrutivos para o módulo de elasticidade (MOE) e módulo de ruptura (MOR) foram determinados conforme a norma ASTM D 143-94 [12]. Para cada tratamento foram utilizados 30 corpos de prova, previamente climatizados.

Testes de homogeneidade de variâncias (teste de Bartlett, a 5\% de significância) e de normalidade (teste Shapiro-Wilk, a 5\% de significância) foram realizados antes da análise de variância. As médias dos tratamentos foram submetidas ao teste de Scott-Knott em nível de 5\% de significância.

\section{RESULTADOS E DISCUSSÃO}

A termorretificação reduziu a massa, umidade de equilíbrio e densidade básica da madeira de Pinus oocarpa e Eucalyptus urophylla (Tabela 1). 
Tabela 1: Perda de massa, umidade de equilíbrio e densidade básica da madeira termorretificada de Pinus oocarpa e Eucalyptus urophylla

\begin{tabular}{cccc}
\hline \multirow{2}{*}{ Tratamento } & \multicolumn{3}{c}{ Pinus oocarpa } \\
\cline { 2 - 4 } & Perda de massa (\%) & Umidade de equilíbrio (\%) & Densidade básica $\left(\mathrm{g} / \mathrm{cm}^{3}\right)$ \\
\hline Testemunha & - & $13,44^{1,6} \mathrm{a}$ & $0,390^{8,6} \mathrm{a}$ \\
$150^{\circ} \mathrm{C}$ & $0,86^{13,5} \mathrm{a}$ & $12,26^{1,8} \mathrm{~b}$ & $0,386^{7,4} \mathrm{a}$ \\
$175^{\circ} \mathrm{C}$ & $2,34^{10,4} \mathrm{~b}$ & $11,21^{1,5} \mathrm{c}$ & $0,398^{6,8} \mathrm{a}$ \\
$200^{\circ} \mathrm{C}$ & $7,99^{7,5} \mathrm{C}$ & $9,18^{1,9} \mathrm{~d}$ & $0,361^{7,2} \mathrm{~b}$ \\
$225^{\circ} \mathrm{C}$ & $13,89^{9,3} \mathrm{~d}$ & $7,39^{1,6} \mathrm{e}$ & $0,355^{9,2} \mathrm{~b}$ \\
\hline \multirow{2}{*}{ Tratamento } & \multicolumn{4}{c}{ Eucalyptus urophylla } \\
\cline { 2 - 4 } & Perda de massa (\%) & Umidade de equilíbrio $(\%)$ & Densidade básica $\left(\mathrm{g} / \mathrm{cm}^{3}\right)$ \\
\hline Testemunha & - & $11,58^{2,1} \mathrm{a}$ & $0,551^{7,8} \mathrm{a}$ \\
$150^{\circ} \mathrm{C}$ & $0,85^{15,4} \mathrm{a}$ & $11,15^{1,4} \mathrm{a}$ & $0,547^{9,1} \mathrm{a}$ \\
$175^{\circ} \mathrm{C}$ & $2,38^{10,0} \mathrm{~b}$ & $9,78^{1,3} \mathrm{~b}$ & $0,5699^{8,8} \mathrm{a}$ \\
$200^{\circ} \mathrm{C}$ & $11,09^{6,6} \mathrm{C}$ & $7,31^{1,7} \mathrm{C}$ & $0,510^{8,3} \mathrm{~b}$ \\
$225^{\circ} \mathrm{C}$ & $15,97^{7,2} \mathrm{~d}$ & $6,66^{1,4} \mathrm{~d}$ & $0,505^{8,8} \mathrm{~b}$ \\
\hline Médias seguidas de mesma letra por coluna em cada espécie não diferem pelo teste de Tukey a 5\% de probabilidade. Valores em sobrescrito representam o coeficiente \\
de variação
\end{tabular}

O incremento da temperatura de termorretificação aumentou exponencialmente a perda de massa. Nas temperaturas mais baixas, a perda de massa ocorreu devido à degradação de extrativos, principalmente os polares [1, 13]. Em temperaturas mais altas ocorreu devido a degradação das hemiceluloses [14,15], presentes em maior proporção na madeira que os extrativos o que explica a maior perda de massa em temperaturas mais elevadas.

A perda de massa foi de $13,89 \%$ e $15,97 \%$ na madeira tratada a $225^{\circ} \mathrm{C}$ para Pinus oocarpa e Eucalyptus urophylla, respectivamente. Isto confirma o fato de, em geral, as coníferas terem maior resistência à degradação térmica e, por isto, maior rendimento quando submetidas à altas temperaturas. Isto foi relatado para madeira termorretificada de Pinus pinaster em relação a Eucalyptus globulus [16] e carvão de Pinus oocarpa em relação a Eucalyptus paniculata e Corymbia citriodora [17].

Tanto para o Pinus oocarpa quanto para o Eucalyptus urophylla, a umidade de equilíbrio foi menor a partir do tratamento a $150^{\circ} \mathrm{C}$, mas ambas as espécies apresentaram maior queda deste parâmetro nos tratamentos com maior temperatura. O tratamento térmico nestas temperaturas reduz grande parte dos sítios hidrofílicos e compromete a capacidade de adsorção de água do ambiente [1, 18], devido a degradação das hemiceluloses [19].

O processo de termorretificação causa perda da massa e contração da madeira [18]. A perda de massa ocorreu em maior proporção do que a contração, desse modo, a madeira termorretificada de Eucalyptus urophylla e Pinus oocarpa apresentou menor densidade a partir do tratamento a $200^{\circ} \mathrm{C}$.

A termorretificação reduziu o inchamento linear e, consequentemente o volumétrico, além do coeficiente de anisotropia (Tabela 2).

Tabela 2: Inchamento volumétrico, tangencial e radial e coeficiente de anisotropia da madeira de Pinus oocarpa e Eucalyptus urophylla termorretificada

\begin{tabular}{|c|c|c|c|c|}
\hline \multirow[b]{2}{*}{ Tratamento } & \multicolumn{4}{|c|}{ Pinus oocarpa } \\
\hline & Inch.volumétrico (\%) & Inch. tangencial (\%) & Inch. radial (\%) & $\begin{array}{c}\text { Coeficiente de } \\
\text { anisotropia }\end{array}$ \\
\hline Testemunha & $13,45^{7,7}$ a & $5,55^{14,5} \mathrm{a}$ & $2,61^{15,8} \mathrm{a}$ & $2,22^{15,6} \mathrm{a}$ \\
\hline $150{ }^{\circ} \mathrm{C}$ & $12,29^{8,3} \mathrm{~b}$ & $5,35^{14,3} \mathrm{a}$ & $2,69^{15,6} a$ & $2,01^{14,8} \mathrm{~b}$ \\
\hline $175{ }^{\circ} \mathrm{C}$ & $11,04^{9,6} \mathrm{C}$ & $4,38^{14,7} b$ & $2,27^{9,3} \mathrm{~b}$ & $1,95^{10,4} \mathrm{~b}$ \\
\hline $200{ }^{\circ} \mathrm{C}$ & $9,58^{9,2} \mathrm{~d}$ & $3,42^{13,6} c$ & $1,94^{14,6} \mathrm{C}$ & $1,82^{13,9} \mathrm{c}$ \\
\hline $225{ }^{\circ} \mathrm{C}$ & $8,59^{8,7}$ e & $2,77^{14,7} \mathrm{~d}$ & $1,61^{14,2} \mathrm{~d}$ & $1,76^{13,1} \mathrm{c}$ \\
\hline \multirow[b]{2}{*}{ Tratamento } & \multicolumn{4}{|c|}{ Eucalyptus urophylla } \\
\hline & Inch.volumétrico (\%) & Inch. tangencial (\%) & Inch. radial (\%) & $\begin{array}{c}\text { Coeficiente de } \\
\text { anisotropia }\end{array}$ \\
\hline Testemunha & $17,83^{4,7} \mathrm{a}$ & $9,22^{14,2} \mathrm{a}$ & $5,64^{7,6}$ a & $1,64^{13,5} \mathrm{a}$ \\
\hline $150{ }^{\circ} \mathrm{C}$ & $16,89^{6,1} \mathrm{~b}$ & $7,95^{11,1} \mathrm{~b}$ & $5,50^{7,2} \mathrm{a}$ & $1,45^{12,4} \mathrm{~b}$ \\
\hline $175{ }^{\circ} \mathrm{C}$ & $15,46^{5,7} \mathrm{c}$ & $7,34^{9,7} \mathrm{c}$ & $5,11^{6,3} \mathrm{~b}$ & $1,44^{9,2} \mathrm{~b}$ \\
\hline $200{ }^{\circ} \mathrm{C}$ & $10,43^{5,9} \mathrm{~d}$ & $4,29^{13,2} d$ & $3,22^{12,9} \mathrm{c}$ & $1,32^{12,3} \mathrm{c}$ \\
\hline $225^{\circ} \mathrm{C}$ & $8,44^{4,9}$ e & $3,62^{10,3} \mathrm{e}$ & $2,82^{14,3} \mathrm{~d}$ & $1,30^{11,6} \mathrm{c}$ \\
\hline
\end{tabular}


O inchamento tangencial foi maior que o radial em todos os tratamentos, semelhante ao relatado para madeira de Corymbia citriodora, Eucalyptus grandis, Eucalyptus saligna e Pinus elliottii [20,21]. Isto ocorre devido à orientação das células do raio, que dispõem suas microfibrilas no sentido radial e oferecendo maior resistência a variações em suas dimensões [22,23].

A madeira de Eucalyptus urophylla apresentou maior inchamento linear e, consequentemente, maior inchamento volumétrico. A maior densidade da madeira resulta em um maior número de sítios hidrofílicos e aumento da adsorção e, com isso, maior inchamento [23,24]. Entretanto, a menor diferença proporcional, entre o inchamento tangencial e radial resultou em um menor coeficiente da anisotropia.

A termorretificação causou maior redução no inchamento tangencial, resultando em queda do coeficiente de anisotropia de $20,7 \%$ para a madeira termorretificada a $225^{\circ} \mathrm{C}$ de $P$. oocarpa e E. urophylla. A redução do coeficiente de anisotropia devido à termorretificação foi, também, relatada para madeira de Pinus pinaster [25] e Eucalyptus globulus [26].

A termorretificação diminuiu a resistência mecânica e, consequentemente, o modulo de elasticidade e de ruptura da madeira de Pinus oocarpa e Eucalyptus urophylla (Tabela 3).

Tabela 3: Modulo de elasticidade e de ruptura da madeira termorretificada de Pinus oocarpa e Eucalyptus urophylla

\begin{tabular}{lll}
\hline \multirow{2}{*}{ Tratamento } & Pinus oocarpa \\
\cline { 2 - 3 } & Modulo de ruptura (MPa) & Modulo de elasticidade (MPa) \\
\hline Testemunha & $66,4^{12,1} \mathrm{a}$ & $2362^{14,3} \mathrm{a}$ \\
$150^{\circ} \mathrm{C}$ & $57,3^{14,6} \mathrm{~b}$ & $2125^{13,4} \mathrm{~b}$ \\
$175^{\circ} \mathrm{C}$ & $42,7^{15,7} \mathrm{C}$ & $2135^{11,8} \mathrm{~b}$ \\
$200^{\circ} \mathrm{C}$ & $30,4^{10,8} \mathrm{~d}$ & $1983^{9,6} \mathrm{C}$ \\
$225^{\circ} \mathrm{C}$ & $24,6^{11,0} \mathrm{e}$ & $1713^{11,7} \mathrm{~d}$ \\
\hline \multirow{2}{*}{ Tratamento } & Eucalyptus urophylla & \\
\cline { 2 - 3 } & Modulo de ruptura (MPa) & Modulo de elasticidade (MPa) \\
\hline Testemunha & $116,2^{12,1} \mathrm{a}$ & $4134^{13,2} \mathrm{a}$ \\
$150^{\circ} \mathrm{C}$ & $92,3^{13,4} \mathrm{~b}$ & $4108^{11,7} \mathrm{a}$ \\
$175^{\circ} \mathrm{C}$ & $83,4^{12,7} \mathrm{C}$ & $3753^{13,2} \mathrm{~b}$ \\
$200^{\circ} \mathrm{C}$ & $56,2^{12,3} \mathrm{~d}$ & $3369^{10,3} \mathrm{C}$ \\
$225^{\circ} \mathrm{C}$ & $49,1^{14,2} \mathrm{e}$ & $3123^{11,7} \mathrm{C}$ \\
\hline Médias seguidas de mesma letra por coluna em cada espécie não diferem pelo teste de Tukey a 5\% de probabilidade. Valores em sobrescrito representam o coeficiente \\
de variação & &
\end{tabular}

A madeira sem termorretificação de Eucalyptus grandis apresentou maior resistência mecânica em relação a de Pinus oocarpa devido a sua maior densidade. Madeiras com maiores valores de densidade apresentam maior massa de material lenhoso por unidade de volume e, portanto, maior modulo de elasticidade e de ruptura $[27,28]$.

A redução do modulo de ruptura da madeira de Pinus oocarpa e Eucalyptus urophylla acompanhou a degradação de seus carboidratos, com redução a partir de $150^{\circ} \mathrm{C}$. Isto ocorre devido a degradação de componentes estruturais da madeira, como as hemiceluloses, implicando em perda da resistência mecânica [29].

A termorretificação diminuiu o modulo de elasticidade da madeira de E. urophylla e de P. oocarpa, principalmente a partir de $200^{\circ} \mathrm{C}$. Tendência semelhante foi encontrada para madeira de Eucalyptus regnans [10] e contrária ao aumento registrado para Eucalyptus globulus [30] enquanto que Eucalyptus grandis e Eucalyptus saligna [31] não apresentaram alteração desta propriedade após termorretificação. Isto evidencia que o comportamento do modulo de elasticidade pode variar com o tratamento térmico, dependendo da madeira utilizada.

A madeira deve, preferencialmente, ter alta estabilidade dimensional e resistência mecânica. Portanto, é necessário, no processo de termorretificação, encontrar o ponto ótimo entre a melhora das propriedades físicas e a redução da resistência mecânica da madeira.

Os resultados para o modulo de elasticidade dinâmico por ressonância através do Bing apresentaram alta relação com os de elasticidade estático, com coeficiente de correlação de 0,8182 e 0,8984 para a madeira de Pinus oocarpa e Eucalyptus urophylla, semelhante ao encontrado para Copaifera langsdorffii, de 0,88 [11]. O modulo de elasticidade estático é diretamente proporcional ao de ruptura [32]. Isto permitiu estimar, também, este último a partir do modulo de elasticidade dinâmico determinado pelo Bing, com coeficiente de determinação de 0,5116 e 0,6004 para Pinus oocarpa e Eucalyptus urophylla, respectivamente, entretando a precisão não foi tão alta, de modo que o resultado mostrado pelo sistema BING e o método convencional apresentou correlação moderada (Figura 1). O desvio entre o determinado pelo método convencional e o encontrado pelo sistema BING apresentaram distribuição normal sem a presença de homocedasticidade, tanto para o modulo de elasticidade quanto para o modulo de ruptura, o que aumentam ainda mais a qualidade dos resultados. 

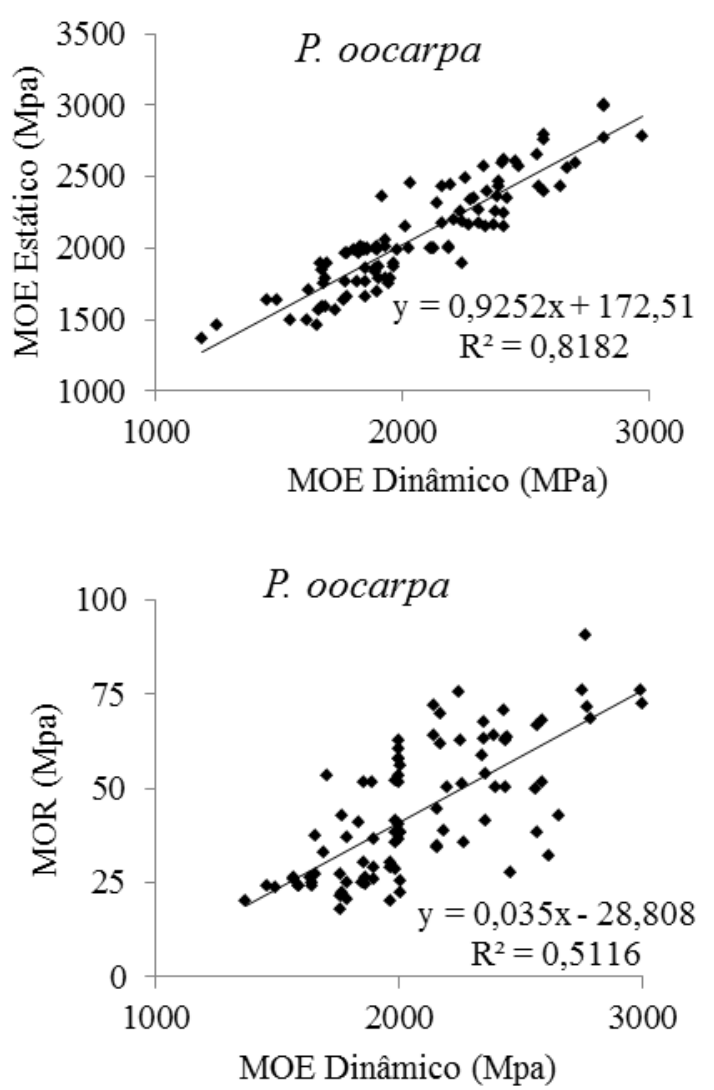
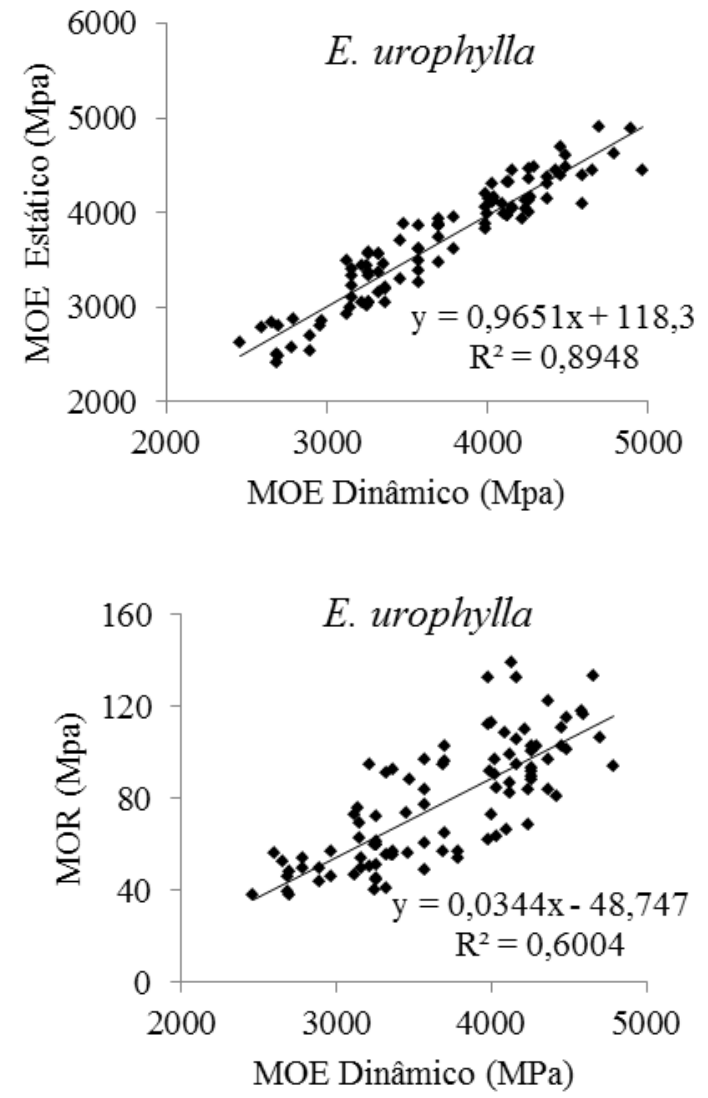

Figura 1: A correlação entre o módulo de elasticidade estático e módulo de ruptura com o módulo de elasticidade dinâmico medido pelo sistema BING na madeira termorretificada de Eucalyptus urophylla e Pinus oocarpa.

A análise de ressonância com o Bing permitiu estimar o modulo de elasticidade e ruptura da madeira termorretificada como uma opção rápida e barata para o controle deste processo.

\section{CONCLUSÕES}

A termorretificação reduziu a umidade de equilíbrio e inchamento volumétrico e linear, o que melhorou as propriedades físicas da madeira de Pinus oocarpa e Eucalyptus urophylla. Entretanto, a aplicação de calor através da termorretificação reduziu o modulo de elasticidade estático e modulo de ruptura da madeira. O modulo de elasticidade dinâmico, determinado por ressonância através do Bing apresentou relação com o modulo de elasticidade estático e modulo de ruptura da madeira termorretificada de Pinus oocarpa e Eucalyptus urophylla, por isso, a analise de ressonância pode ser uma alternativa para determinação destas propriedades mecânicas de maneira rápida, barata e não destrutiva na madeira termorretificada.

\section{AGRADECIMENTOS}

A Coordenação de Aperfeiçoamento de Pessoal de Nível Superior (CAPES), Conselho Nacional de Desenvolvimento Científico e Tecnológico $(\mathrm{CNPq})$ e Fundação de Amparo à Pesquisa do Estado de Minas Gerais (Fapemig).

\section{BIBLIOGRAFIA}

[1] ESTEVES, B. M., PEREIRA, H. M. Wood modification by heat treatment: A review, BioResources, v. 4, n. 1, pp. 370-404, 2009.

[2] YALCIN, M., SAHIN, H. I. "Changes in the chemical structure and decay resistance of heat-treated narrow-leaved ash wood”, Maderas- Ciencia y Tecnologia, v.7, pp.435-446, 2015.

[3] KESIK, H. I., KORKUT, S., HIZIROGLU, S., et al. "An evaluation of properties of four heat treated wood species”, Industrial Crops and Products, v.60, pp.60-65, 2014. 
[4] ARAÚJO, S. O., VITAL, B. R., DE MENDOZA, Z. M. S. H., et al. "Propriedades de madeiras termorretificadas de Eucalyptus grandis e SP”, Scientia Forestalis, v. 40, n. 95, pp. 327-336, set. 2012.

[5] OUMAROU, N., KOCAEFE, D., KOCAEFE, Y. "Some investigations on moisture injection, moisture diffusivity and thermal conductivity using a three-dimensional computation of wood heat treatment at high temperature”, International Communications in Heat And Mass Transfer, v.61, pp.153-161, 2015.

[6] KORKUT, S. "Performance of three thermally treated tropical wood species commonly used in Turkey", Industrial Crops and Products, v.36, pp.355-362, 2012.

[7] BRITO, J. O., SILVA, F. G., LEÃO, M. M., et al. "Chemical composition changes in Eucalyptus and Pinus woods submitted to heat treatment”, Bioresource Technology, v. 99, n. 18, pp. 8545-8548, 2008.

[8] SEVERO, E. T. D., CALONEGO, F. W., SANSIGOLO, C. A. "Physical and chemical changes in juvenile and mature woods of Pinus elliottii var. elliottii by thermal modification", European Journal of Wood and Wood Products, v.70, pp.741-747, 2012.

[9] TIRYAKI, S., HAMZAÇEBI, A. "Predicting modulus of rupture (MOR) and modulus of elasticity (MOE) of heat treated woods by artificial neural networks", Measurement, v.49, pp.266-274, 2014.

[10] ZHANG, T. T., TU, D. Y., PENG, C., et al. "Effects of heat treatment on physical-mechanical properties of Eucalyptus regnans”, BioResources, v.10, pp.3531-3540, 2015.

[11] LEITE, E. R. S., HEIN, P. R. G., SOUZA, T. M., et al., "Estimation of the dynamic elastic properties of wood from Copaifera langsdorffii Desf using resonance analysis”, Cerne, v.18, n.1, pp. 41-47, 2012.

[12] AMERICAN SOCIETY FOR TESTING AND MATERIALS- ASTM. 1997. Standard methods of testing small, clear specimens of timber. D 143-94: pp. 23-53 in Annual book of ASTM standards. Denvers: 1997, 679 p.

[13] MÉSZÁROS, E., JAKAB, E., VÁRHEGYI, G. TG/ MS, "Py-GC/MS and THM-GC/MS study of the composition and thermal behavior of extractive components of Robinia pseudoacacia", Journal of Analytical and Applied Pyrolysis, v.79, n.1, pp.61-70, 2007.

[14] CADEMARTORI, P. H. G., SANTOS, P. S. B., SERRANO L., et al. "Effect of thermal treatment on physicochemical properties of Gympie messmate wood", Industrial Crops and Products, v. 45, pp. 360-366, 2013.

[15] ZANUNCIO, A. J. V., NOBRE, J. R. C., MOTTA, J. P., et al., "Componentes Químicos e colorimétricos da madeira de Eucalyptus grandis”, Revista Árvore, v.38, n. 4, pp. 563-571, 2014.

[16] ESTEVES, B., MARQUES, A.V., DOMINGOS, I. et al, "Chemical changes of heat treated pine and eucalypt wood monitored by ftir. Maderas”, Ciencia y Tecnología, v.15, n.2, pp. 245-258, 2013.

[17] ZANUNCIO, A.J.V., CARVALHO, A. G., TRUGILHO, P. F., et al. "Extractives and energetic properties of wood and charcoal”, Revista Árvore, v.38, n.2, pp.369-374, 2014.

[18] ZANUNCIO, A. J. V., MOTTA, J. P., SILVEIRA, T. A., et al., "Physical and colorimetric changes in Eucalyptus grandis wood after heat treatment”, BioResources, v.9, pp.293-302, 2014.

[19] POLETTO, A, M., ZATTERA, A. J., FORTEA, M. M. C., et al. "Thermal decomposition of wood: Influence of wood components and cellulose crystallite size”, Bioresource Technology, v. 109, pp. 148-153, April 2012.

[20] MENEZES, W. M., SANTINI, E. J., SOUZA, J. T., et al. "Modificação térmica nas propriedades físicas da madeira”, Ciência Rural, v.44, n.6, pp.1019-1024, jun, 2014.

[21] PELOZZI, M. M. A., SEVERO, E. T. D., CALONEGO, F. W., et al. "Propriedades físicas dos lenhos juvenil e adulto de Pinus elliottii Engelm var. elliottii e de Eucalyptus grandis Hill ex Maiden”, Ciência Florestal, v. 22, n. 2, pp. 305-313, abr.-jun., 2012

[22] KOLLMANN, F. F. P., CÔTÉ, W. A. Principles of wood science and technology, solid wood, 1 ed., New York, Springer, 1968.

[23] SCHULGASSER, K., WITZTUM, A. "How the relationship between density and shrinkage of wood depends on its microstructure”, Wood Science and Technology, v. 49, n. 2, pp. 389-401, 2015.

[24] ROUCO, M. C. A,, MUNOZ, G. R. "Influence of blue stain on density and dimensional stability of Pinus radiata timber from northern Galicia (Spain)”, Holzforschung, v. 69, n. 1, pp. 97-102, 2015.

[25] ESTEVES, B. M., DOMINGOS, I. J., PEREIRA, H. M. "Pine wood modification by heat treatment in air”, BioResources, v.3, n.1, pp.142-154, 2008. 
[26] ESTEVES, B., DOMINGOS, I., PEREIRA, H. "Improvement of technological quality of eucalypt wood by heat treatment in air at 170-200 ${ }^{\circ}$ ”, For. Prod. J., v. 57, n.1/2, pp.47-52, 2007.

[27] NIKLAS, K. J., SPATZ, H.C. "Worldwide correlations of mechanical properties and green wood density”, American Journal of Botany, v. 97, n. 10, pp. 1587-94, 2010.

[28] NIKLAS, K. J., SPATZ, H.C. "Mechanical properties of wood disproportionately increase with increasing density”, American Journal of Botany, v.99, n.1, pp.169-70, 2012.

[29] KACIKOVA, D., KACIK, F., CABALOVA, I., et al. "Effects of thermal treatment on chemical, mechanical and colour traits in Norway spruce wood”, Bioresource Technology, v. 144, pp. 669-674, 2013.

[30] SANTOS, J. "Mechanical behaviour of Eucalyptus wood modified by heat”, Wood Science and Technology, v.34, pp.39-43, 2000.

[31] CADEMARTORI, P. H. G., MISSIO, A. L., MATTOS, B. D., et al., "Effect of thermal treatments on technological properties of wood from two Eucalyptus species”, Anais da Academia Brasileira de Ciencias, v.87, pp.471-481, 2015.

[32] LOURENÇON, T. V., MATTOS, B. D., GATTO, D. A., et al., "Determinação da idade de transição entre lenho juvenil e lenho adulto para três espécies florestais por meio de suas propriedades mecânicas”, Floresta e Ambiente, v. 21, n.2, pp.251-260, abr./jun2014. 